Publ. Mat. 58 (2014), 499-516

DOI: 10.5565/PUBLMAT_58214_25

\title{
EMBEDDINGS OF LOCAL FIELDS IN SIMPLE ALGEBRAS AND SIMPLICIAL STRUCTURES
}

\author{
DANIEL SKODLERACK
}

\begin{abstract}
We give a geometric interpretation of Broussous-Grabitz embedding types. We fix a central division algebra $D$ of finite index over a non-Archimedean local field $F$ and a positive integer $m$. Further we fix a hereditary order $\mathfrak{a}$ of $\mathrm{M}_{m}(D)$ and an unramified field extension $E \mid F$ in $\mathrm{M}_{m}(D)$ which is embeddable in $D$ and which normalizes $\mathfrak{a}$. Such a pair $(E, \mathfrak{a})$ is called an embedding. The embedding types classify the $\mathrm{GL}_{m}(D)$-conjugation classes of these embeddings. Such a type is a class of matrices with non-negative integer entries. We give a formula which allows us to recover the embedding type of $(E, \mathfrak{a})$ from the simplicial type of the image of the barycenter of $\mathfrak{a}$ under the canonical isomorphism, from the set of $E^{\times}$-fixed points of the reduced building of $\mathrm{GL}_{m}(D)$ to the reduced building of the centralizer of $E^{\times}$ in $\mathrm{GL}_{m}(D)$. Conversely the formula allows to calculate the simplicial type up to cyclic permutation of the Coxeter diagram.
\end{abstract}

2010 Mathematics Subject Classification: 17C20, 20E42, 12J25.

Key words: Embeddings types, buildings, simple algebras, non-archimedean local fields.

\section{Introduction}

Field extensions in Azumaya algebras with respect to local representation theory have been studied in many situations. We want to mention works of Fröhlich [Frö87], Broussous and Grabitz [Gra99], [BG00] among others. More precisely we consider a skew field $D$ of finite index $d$ over a non-Archimedean local field $F$ and pairs $(E, \mathfrak{a})$ consisting of a field extension $E \mid F$ in $\mathrm{M}_{m}(D)$ and a hereditary order $\mathfrak{a}$ of $\mathrm{M}_{m}(D)$ normalized by $E^{\times}$. Such a pair $(E, \mathfrak{a})$ is called an embedding. In the representation theory of $G:=\mathrm{GL}_{m}(D)$ on complex vector spaces it has been important to understand when two such embeddings are conjugate to each other under $G$. Broussous and Grabitz attached in [BG00, 2.3.10, 2.3.1] to every embedding a cyclic permutation class of matrices with non-negative integer entries, which only depends on $\mathfrak{a}$ and the maximal unramified field extension $E_{D} \mid F$ of $E \mid F$ embeddable in $D \mid F$. We call the latter class embedding type of $(E, \mathfrak{a})$. It is slightly different to the 
"type of embedding" in their paper, where they restrict to matrices with $d$ rows. This invariant allows to define certain equivalent embeddings, called pearl embeddings, which are suitable for calculations and which are conjugate to $\left(E_{D}, \mathfrak{a}\right)$. The latter is a generalization of a work [Frö87] of Fröhlich, where he considered the case of principal orders. Broussous and Grabitz proved:

Proposition 1.1 ([BG00, 3.2]). Two embeddings $(E, \mathfrak{a})$ and $\left(E^{\prime}, \mathfrak{a}\right)$ are conjugate under $\mathrm{GL}_{m}(D)$ if and only if the field extensions are isomorphic and the embeddings have the same embedding type, i.e. $\left(E_{D}, \mathfrak{a}\right)$ and $\left(E_{D}^{\prime}, \mathfrak{a}\right)$ are conjugate under $\mathrm{GL}_{m}(D)$.

This rigidity statement plays a role in the classification of supercuspidal representations of $G$, see for example [BSS12].

The main result of this paper is that the embedding type can be obtained geometrically. The strategy is the following. We denote by $G_{E_{D}}$ the centralizer of $E_{D}$ in $G$. Broussous and Lemaire introduced a canonical isomorphism between reduced Bruhat-Tits buildings

$$
j_{E_{D}}: \mathfrak{B}_{\text {red }}(G)^{E_{D}^{\times}} \rightarrow \mathfrak{B}_{\text {red }}\left(G_{E_{D}}\right)
$$

which is affine, $G_{E_{D}}$-equivariant and which respects the Moy-Prasad filtrations [MP94]. Let $(E, \mathfrak{a})$ be an embedding. The barycenter $x_{\mathfrak{a}}$ of $\mathfrak{a}$ in $\mathfrak{B}_{\text {red }}(G)$ is fixed under the action of $E_{D}^{\times}$.

In this article we construct an easy combinatorial map ()$^{c}$ of order two on the set of non-zero cyclic vectors with non-negative integer coefficients. A cyclic vector $\langle v\rangle$ is the set of vectors which are equal to $v$ up to cyclic permutation of the coordinates. We prove:

Theorem 1.2 (see Theorem 6.4). Let $M$ be an element of the embedding type of $(E, \mathfrak{a})$, in particular $M$ is a matrix with non-negative integer coefficients. Write $M$ line-wise into a vector $\lambda$. Fix a labeling of $\mathfrak{B}_{\text {red }}\left(G_{E_{D}}\right)$ and let $\mu$ be the barycentric coordinates of $j_{E_{D}}\left(x_{\mathfrak{a}}\right)$. Then $\left(\left\langle\operatorname{rank}(\mathfrak{a})\left[E_{D}: F\right] \mu\right\rangle\right)^{c}$ is equal to $\langle\lambda\rangle$.

Here $\operatorname{rank}(\mathfrak{a})$ is the simplicial rank of $\mathfrak{a}$ seen as a facet of $\mathfrak{B}_{\text {red }}(G)$, see Definition 2.5. The advantage of this approach is the following. On can consider classes of embeddings as classes of certain points with rational coordinates in a chamber of $\mathfrak{B}_{\text {red }}\left(G_{E_{D}}\right)$. The class is given by the action of the group of rotations of the Coxeter diagram on $\mathfrak{B}_{\text {red }}\left(G_{E_{D}}\right)$. Let $h$ be a Hermitian form on $D^{m}$ and $\mathrm{U}(h)$ its group of isometries. One can now study examples of embeddings which are invariant under the adjoint involution of $h$ in studying certain points of $j_{E_{D}}\left(\mathfrak{B}(\mathrm{U}(h)) \cap \mathfrak{B}_{\text {red }}(G)^{E_{D}^{\times}}\right)$ with rational coordinates. 
The article is structured as follows. We give general notation and we recall the model of the reduced Bruhat-Tits building of $\mathrm{GL}_{m}(D)$ in terms of lattice functions in $\S 2$ and introduce in $\S 3$ cyclic vectors and matrices. In $\S 4$ we recall definitions and statements of [BG00] on embedding types and pearl embeddings. In relevant cases we recall the description of the map $j_{E}$ in $\S 5$. Finally, $\S 6$ explains the strategy to encode the embedding type from barycentric coordinates, followed by the proof of Theorem 6.4. The proof consists of three main ideas:

1. changing the skew field in Lemma 6.8,

2. a rank reduction for the considered facet $\mathfrak{a}$ in Lemma 6.7, and

3. the proof for the case that $\mathfrak{a}$ is a vertex.

I have very much to thank Professor Zink from Humboldt University Berlin for his helpful remarks, the revision of the work, and for giving me the interesting problem.

\section{The reduced Bruhat-Tits building of $\mathrm{GL}_{m}(D)$}

Let $(F, \nu)$ be a non-Archimedean local field with normalized valuation $\nu$, valuation ring $o_{F}$, valuation ideal $\mathfrak{p}_{F}$, a chosen uniformizer $\pi_{F}$, and residue field $\kappa_{F}$. We use similar notation for other skew fields with non-Archimedean valuation. We fix a skew field $D$ of finite index $d$ and central over $F$, together with a maximal unramified field extension $L \mid F$ in $D \mid F$, and a uniformizer $\pi_{D}$ which normalizes $L$ such that the map

$$
x \mapsto \sigma(x):=\pi_{D} x \pi_{D}^{-1}, \quad x \in D,
$$

generates $\operatorname{Gal}(L \mid F)$. For a positive integer $f \mid d$ we denote by $L_{f}$ the subfield of degree $f$ over $F$ in $L$. Further, let $V$ be a right $D$-vector space of finite dimension $m$ and denote its ring of $D$-linear endomorphisms by $A$. Then $V$ is in a natural way a left $A \otimes_{F} D^{o p}$-module. We write $G$ for $A^{\times}$.

We recall the model of the reduced Bruhat-Tits building $\mathfrak{B}_{\text {red }}(G)$ in terms of lattice functions. For more details we recommend [BL02] and [BT84]. We adopt the following definitions from [BL02].

Definition 2.1. $\quad$ 1. An $m$-dimensional free $o_{D}$-submodule of $V$ is an $o_{D}$-lattice. We denote the set of all $o_{D}$-lattices by $\mathcal{L}\left(o_{D}, V\right)$. It is partially ordered by inclusion.

2. A strictly decreasing map $\Lambda$ from $\mathbb{Z}$ to $\mathcal{L}\left(o_{D}, V\right)$ is called an $o_{D}$-lattice chain on $V$ if there is an $r \in \mathbb{N}$ such that $\mathcal{L}_{i} \pi_{D}=\mathcal{L}_{i+r}, i \in \mathbb{Z}$. Two lattice chains $\mathcal{L}$ and $\mathcal{L}^{\prime}$ are $\mathbb{Z}$-translations of each other if there is an integer $m$ such that $\mathcal{L}_{i+m}$ is equal to $\mathcal{L}_{i}$, for all integers $i$. A translation class is denoted by $[\mathcal{L}]$. 
3. A decreasing map $\Lambda$ from $\mathbb{R}$ to $\mathcal{L}\left(o_{D}, V\right)$ is called an $o_{D}$-lattice function on $V$ if it is

- left continuous, i.e. $\Lambda(t)=\cap_{s<t} \Lambda(s)$, and

- satisfies $\Lambda(t) \pi_{D}=\Lambda\left(t+\frac{1}{d}\right), t \in \mathbb{R}$.

The set of $o_{D}$-lattice functions is denoted by $\operatorname{Latt}_{o_{D}}^{1}(V)$. The map which sends $\Lambda$ to $(\Lambda(t-s))_{t \in \mathbb{R}}$, which we denote by $\Lambda+s$, is called a translation by $s$. Two $o_{D}$-lattice functions are equivalent if they differ by a translation and the set of all classes of $o_{D}$-lattice functions is denoted by $\operatorname{Latt}_{o_{D}}(V)$. For $[\Lambda] \in \operatorname{Latt}_{o_{D}}(V)$, the square lattice function of $\Lambda$ is defined to be the following $o_{F}$-lattice function in $A$ :

$$
t \mapsto \mathfrak{g}_{\Lambda}(t):=\{a \in A \mid a(\Lambda(s)) \subseteq \Lambda(s+t) \text { for all } s \in \mathbb{R}\}
$$

This attachment only depends on the translation class of $\Lambda$ and is injective on $\operatorname{Latt}_{o_{D}}(V)$, i.e. two different translation classes have different square lattice functions, see $[$ BL02]. We denote the set of square lattice functions of $A$ by $\operatorname{Latt}_{O_{F}}^{2}(A)$.

4. The hereditary order $\mathfrak{a}_{\Lambda}$ corresponding to $\Lambda$ is the ring $\mathfrak{g}_{\Lambda}(0)$, which only depends on the translation class of $\Lambda$.

Theorem 2.2 ([BL02, I 2.4]). There is a unique G-equivariant, affine bijection from $\mathfrak{B}_{\text {red }}(G)$ to $\operatorname{Latt}_{o_{D}}(V)$.

Remark 2.3. 1. The apartments of $\mathfrak{B}_{\text {red }}(G)$ carry over to $\operatorname{Latt}_{o_{D}}(V)$; more precisely the set of apartments in $\operatorname{Latt}_{o_{D}}(V)$ is in one to one correspondence with the set of frames in $V$. A frame is a set of one dimensional $D$-sub-vector spaces of $V$ whose direct sum is $V$. The apartment corresponding to a frame $\mathcal{R}$ is the set $\operatorname{Latt}_{\mathcal{R}}(V)$ of $[\Lambda]$ such that $\Lambda$ is split by $\mathcal{R}$, i.e.

$$
\Lambda(t)=\oplus_{W \in \mathcal{R}} \Lambda(t) \cap W,
$$

for all $t \in \mathbb{R}$.

2. The inherited simplicial structure on $\operatorname{Latt}_{o_{D}}(V)$ is given as follows:

The facet containing $[\Lambda] \in \operatorname{Latt}_{O_{D}}(V)$ is

$$
\left\{\left[\Lambda^{\prime}\right] \in \operatorname{Latt}_{o_{D}}(V) \mid \operatorname{im}(\Lambda)=\operatorname{im}\left(\Lambda^{\prime}\right)\right\} .
$$

Theorem 2.4 ([Rei03, 39.14]). There are canonical bijections between the set of $\mathbb{Z}$-translation classes of $o_{D}$-lattice chains in $V$, the set of images of elements of $\operatorname{Latt}_{o_{D}}^{1}(V)$, and the set of hereditary orders in $A$ :

$$
[\mathcal{L}] \mapsto \operatorname{im}(\mathcal{L}) \text { and } \operatorname{im}(\Lambda) \mapsto \mathfrak{a}_{\Lambda} .
$$


To be parallel to the notation in [BL02] we write $\mathcal{I}$ for $\mathfrak{B}_{\text {red }}(G)$ and identify it with $\operatorname{Latt}_{o_{D}}(V)$. Its simplicial structure is denoted by $\Omega$. We also call $\Omega$ the Euclidean building of $G$, so in this article a Euclidean building is a simplicial complex and not its geometric realization.

The simplices are denoted by hereditary orders, i.e. the simplex of $x \in$ $\mathcal{I}$ is denoted by $\mathfrak{a}_{x}$. The square lattice function attached to $x$ is denoted by $\mathfrak{g}_{x}$.

For a frame $\mathcal{R}$, we denote the appartment of $\mathcal{I}$ corresponding to $\mathcal{R}$ by $\mathcal{I}_{\mathcal{R}}$ and its simplicial structure by $\Omega_{\mathcal{R}}$, more precisely we have

$$
\Omega_{\mathcal{R}}=\left\{\mathfrak{a} \in \Omega \mid \exists[\Lambda] \in \operatorname{Latt}_{\mathcal{R}}(V): \mathfrak{a}=\mathfrak{a}_{\Lambda}\right\} .
$$

We also call $\Omega_{\mathcal{R}}$ the apartment of $\Omega$ corresponding to $\mathcal{R}$, i.e. we have apartments for $\mathcal{I}$ and for $\Omega$.

Definition 2.5. Given two simplices $\mathfrak{a}$ and $\mathfrak{a}^{\prime}$, we write $\mathfrak{a} \leq \mathfrak{a}^{\prime}$ if $\mathfrak{a} \supseteq \mathfrak{a}^{\prime}$. A vertex of $\Omega$ is a simplex which is minimal with respect to $\leq$. Let $\mathfrak{b}$ be a vertex of $\Omega$ and $\mathfrak{a}$ a simplex. We call $\mathfrak{b}$ a vertex of $\mathfrak{a}$ if $\mathfrak{b} \leq \mathfrak{a}$. We define the simplicial rank of $\mathfrak{a}$ as the number of vertices of $\mathfrak{a}$, and denote it by $\operatorname{rank}(\mathfrak{a})$. A simplex of maximal rank is a chamber of $\Omega$.

\section{Cyclic vectors and matrices}

The invariants which are considered in this article are vectors or matrices modulo cyclic permutation.

\subsection{Vectors.}

Definition 3.1. Let $R$ be an arbitrary non-empty set, $\operatorname{Vec}(R)$ be the set of finite dimensional row-vectors with entries in $R$, i.e.

$$
\operatorname{Vec}(R):=\bigcup_{i=1}^{\infty} R^{1 \times i} .
$$

We call two elements $w, w^{\prime}$ of $\operatorname{Vec}(R)$ equivalent if they have the same dimension, say $s$, and if there is a $k$ such that

$$
w^{\prime}=\left(w_{k}, \ldots, w_{s}, w_{1}, \ldots, w_{k-1}\right) .
$$

The equivalence class is denoted by $\langle w\rangle$ and it is a called a cyclic vector. We often skip the round brakets and write $\left\langle w_{1}, \ldots, w_{k}\right\rangle$. The set of equivalence classes of $\operatorname{Vec}(R)$ is denoted by $\operatorname{CVec}(R)$.

We now represent a cyclic vector with entries in $\mathbb{N}_{0}$ in a different way, to be able to attach a dual cyclic vector. There is a canonical map from 
$\operatorname{CVec}\left(\mathbb{N}_{0}\right)$ to $\mathbb{N}_{0}$ which maps a cyclic vector to its sum of the coordinates, and we write $\operatorname{CVec}\left(\mathbb{N}_{0}\right)_{+}$for the preimage of $\mathbb{N}$. Consider the map

$$
\text { pairs: } \operatorname{CVec}\left(\mathbb{N}_{0}\right)_{+} \rightarrow \operatorname{CVec}\left(\mathbb{N}^{2}\right),
$$

defined via

$$
\operatorname{pairs}(\langle w\rangle):=\left\langle\left(w_{i_{0}}, i_{1}-i_{0}\right),\left(w_{i_{1}}, i_{2}-i_{1}\right), \ldots,\left(w_{i_{k}}, i_{0}+s-i_{k}\right)\right\rangle,
$$

where $\left(w_{i_{j}}\right)_{0 \leq j \leq k}$ is the subsequence of the non-zero coordinates.

Lemma 3.2. The map pairs is bijective.

Proof: By the definition of pairs we can rebuild $\langle w\rangle$ directly from pairs $(\langle w\rangle)$ and thus pairs is injective. The preimage of an element

$$
\left\langle\left(a_{0}, b_{0}\right),\left(a_{1}, b_{1}\right),\left(a_{2}, b_{2}\right), \ldots,\left(a_{k}, b_{k}\right)\right\rangle
$$

of $\operatorname{CVec}\left(\mathbb{N}^{2}\right)$ contains the cyclic class of the vector $w=\left(w_{i}\right)_{1 \leq i \leq \sum_{l=0}^{k} b_{l}}$ defined via

$$
w_{i}=\left\{\begin{array}{ll}
a_{j}, & \text { if } i=1+\sum_{l=0}^{j} b_{l} \\
0, & \text { else }
\end{array} .\right.
$$

Thus pairs is surjective.

On $\operatorname{CVec}\left(\mathbb{N}^{2}\right)$ there is a canonical bijection $\mathrm{T}$ which maps an element

$$
\left\langle\left(a_{0}, b_{0}\right),\left(a_{1}, b_{1}\right),\left(a_{2}, b_{2}\right), \ldots,\left(a_{k}, b_{k}\right)\right\rangle
$$

to

$$
\left\langle\left(b_{0}, a_{1}\right),\left(b_{1}, a_{2}\right),\left(b_{2}, a_{3}\right), \ldots,\left(b_{k}, a_{0}\right)\right\rangle,
$$

and it induces the bijection ()$^{c}:=$ pairs $^{-1} \circ \mathrm{T} \circ$ pairs of $\operatorname{CVec}\left(\mathbb{N}_{0}\right)_{+}$. We call $\langle w\rangle^{c}$ the dual of $\langle w\rangle$.

\subsection{Matrices.}

For $r, s, t \in \mathbb{N}, \mathrm{M}_{r, s}(t)$ denotes the set of $r \times s$-matrices with nonnegative integer entries, such that

- in every column there is an entry greater than zero, and

- the sum of all entries is $t$.

For a matrix $M=\left(m_{i, j}\right) \in \mathrm{M}_{r, s}(t)$, we define the vector $\operatorname{row}(M) \in \mathbb{N}_{0}^{1 \times r s}$ to be

$$
\left(m_{1,1}, m_{1,2}, \ldots, m_{1, s}, m_{2,1}, \ldots, m_{2, s}, \ldots, m_{r, 1}, \ldots, m_{r, s}\right) .
$$

Two matrices $M, N \in \mathrm{M}_{r, s}(t)$ are said to be equivalent if $\operatorname{row}(M)$ and $\operatorname{row}(N)$ are. The equivalence class, denoted by $\langle M\rangle$, is called a cyclic matrix. 


\section{Example 3.3.}

$$
\left(\begin{array}{ll}
2 & 0 \\
1 & 3 \\
0 & 1
\end{array}\right) \backsim\left(\begin{array}{ll}
1 & 2 \\
0 & 1 \\
3 & 0
\end{array}\right)
$$

\section{Embedding types}

For a field extension $E \mid F$ we denote by $E_{D} \mid F$ the maximal field extension in $E \mid F$, which is $F$-algebra isomorphic to a subfield of $L$. Its degree is the greatest common divisor of $d$ and the inertia degree of $E \mid F$.

Definition 4.1. An embedding is a pair $(E, \mathfrak{a})$ satisfying

1. $E$ is a field extension of $F$ in $A$,

2. $\mathfrak{a}$ is a hereditary order of $A$, normalized by $E^{\times}$.

Two embeddings $(E, \mathfrak{a})$ and $\left(E^{\prime}, \mathfrak{a}^{\prime}\right)$ are said to be equivalent if there is an element $g \in G$, such that $g E_{D} g^{-1}=E_{D}^{\prime}$ and $g \mathfrak{a} g^{-1}=\mathfrak{a}^{\prime}$.

Remark 4.2. In each equivalence class of embeddings there is a pair such that the field can be embedded in $L$.

Until the end of this section we fix a $D$-basis of $V$ and identify $A$ with $\mathrm{M}_{m}(D)$.

Definition 4.3. Let $f \mid d$ and $r \leq m$. A matrix with $f$ rows and $r$ columns is called an embedding datum if it belongs to $\mathrm{M}_{f, r}(m)$. Given an embedding datum $\lambda=\left(\lambda_{i, j}\right)_{1 \leq i \leq f, 1 \leq j \leq r}$, we define the pearl embedding as follows. The pearl embedding of $\lambda$ (with respect to the fixed $D$-basis of $V)$ is the embedding $\left(E_{\lambda}, \mathfrak{a}_{\lambda}\right)$, which satisfies the following conditions:

1. $E_{\lambda}$ is the image of the monomorphism

$$
x \in L_{f} \mapsto \operatorname{diag}\left(D_{1}(x), D_{2}(x), \ldots, D_{r}(x)\right) \in \mathrm{M}_{m}(D),
$$

where

$$
D_{j}(x)=\operatorname{diag}\left(\sigma^{0}(x) \mathbb{1}_{\lambda_{1, j}}, \sigma^{1}(x) \mathbb{1}_{\lambda_{2, j}}, \ldots, \sigma^{f-1}(x) \mathbb{1}_{\lambda_{f, j}}\right)
$$

and $\mathbb{1}_{k}$ is the identity matrix with $k$ rows, in particular $E_{\lambda} \mid F$ is unramified of degree $f$.

2. $\mathfrak{a}$ is a hereditary order in standard form according to the partition $m=n_{1}+\cdots+n_{r}$ where $n_{j}:=\sum_{i=1}^{f} \lambda_{i, j}$, i.e. $\mathfrak{a}$ is the set of block matrices such that the $(i, j)$-th block has size $n_{i} \times n_{j}$ and has all its entries in $o_{D}$ if $i \geq j$ and in $\mathfrak{p}_{D}$ if $i<j$, respectively. 
Theorem 4.4 ([BG00, 2.3.3 and 2.3.10]). $\quad$ 1. Two pearl embeddings are equivalent if and only if the embedding data are equivalent.

2. In every class of embeddings there is a pearl embedding.

Definition 4.5. Let $(E, \mathfrak{a})$ be an embedding. It is equivalent to a pearl embedding $\left(E_{\lambda}, \mathfrak{a}_{\lambda}\right)$, by the above theorem. The cyclic matrix $\langle\lambda\rangle$ is called the embedding type of $(E, \mathfrak{a})$. This definition does not depend on the choice of the basis by the Skolem-Noether Theorem. If $(E, \mathfrak{a})$ has embedding type $\langle\lambda\rangle$ with $\lambda \in \mathrm{M}_{f, r}(m)$, then $r=\operatorname{rank}(\mathfrak{a})$ and $f=\left[E_{D}: F\right]$.

\section{The map $j_{E}$}

Notation 5.1. For this section let $E \mid F$ be a field extension in $A$ and we set $A_{E}$ to be the centralizer of $E$ in $A$, i.e.

$$
A_{E}:=\mathrm{Z}_{A}(E):=\{a \in A \mid a b=b a \forall b \in E\} .
$$

We denote the Euclidean building of $A_{E}^{\times}$by $\Omega_{E}$ and its geometric realization by $\mathcal{I}_{E}$.

The next results are taken from [BL02].

Theorem 5.2 ([BL02, Theorem II 1.1.]). There exists a unique map

$$
j_{E}: \mathcal{I}^{E^{\times}} \rightarrow \mathcal{I}_{E}
$$

such that for any $x \in \mathcal{I}^{E^{\times}}$and $t \in \mathbb{R}$, we have $\mathfrak{g}_{j_{E}(x)}(t)=A_{E} \cap \mathfrak{g}_{x}(e(E \mid F) t)$. The map $j_{E}$ satisfies the following properties:

1. it is bijective,

2. it is $A_{E}^{\times}$-equivariant, and

3. it is affine.

Moreover its inverse $j_{E}^{-1}$ is the only map $\mathcal{I}_{E} \rightarrow \mathcal{I}$ such that 2 and 3 hold.

We briefly give Broussous and Lemaire's description of $j_{E}$ in terms of lattice functions but only in the case where $E \mid F$ is isomorphic to a sub-extension $L_{f} \mid F$ of $L \mid F$. Then $E \otimes_{F} L \cong \bigoplus_{k=0}^{f-1} L$ coming from the decomposition $1=\sum_{k=0}^{f-1} 1_{k}$ labeled such that the $\operatorname{Gal}(L \mid F)$-action to the second factor gives $\sigma\left(1_{k}\right)=1_{k-1}$ for $k \geq 1$ and $\sigma\left(1_{0}\right)=1_{f-1}$. Applying it on the $E \otimes_{F} L$-module $V$, we get $V=\bigoplus_{k} V_{k}$, where $V_{k}:=1_{k} V$.

Remark 5.3. In this situation, $A_{E} \cong \operatorname{End}_{\mathrm{Z}_{D}\left(L_{f}\right)}\left(V_{0}\right) \cong \mathrm{M}_{m}\left(\mathrm{Z}_{D}\left(L_{f}\right)\right)$. 
Theorem 5.4 ([BL02, II 3.1.]). In terms of lattice functions, $j_{E}$ satisfies $j_{E}^{-1}([\Theta])=[\Lambda]$, with

$$
\Lambda(s):=\bigoplus_{k=0}^{f-1} \Theta\left(s-\frac{k}{d}\right) \pi_{D}^{k}, \quad s \in \mathbb{R},
$$

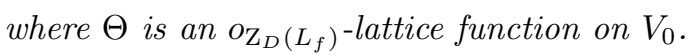

\section{Embedding types through barycentric coordinates}

In this section we keep the notation of Section 5. We need a notion of orientation on $\Omega_{E_{D}}$ to order the barycentric coordinates of a point in $\mathcal{I}_{E_{D}}$.

Definition 6.1. Let $e$ and $e^{\prime}$ be vertices of $\Omega$ joined by an edge, and let $\mathcal{L}$ and $\mathcal{L}^{\prime}$ lattice chains corresponding to $e$ and $e^{\prime}$, respectively. The edge between $e$ and $e^{\prime}$ is oriented towards $e^{\prime}$, if there are lattices $\Gamma \in \operatorname{im}(\mathcal{L})$ and $\Gamma^{\prime} \in \operatorname{im}\left(\mathcal{L}^{\prime}\right)$, such that $\Gamma \supseteq \Gamma^{\prime}$ with the quotient having $\kappa_{D^{-}}$-dimension 1 , i.e. $\kappa_{F}$-dimension $d$. We write $e \rightarrow e^{\prime}$. If $x$ is a point in $\mathcal{I}$ then there is a chamber $C \in \Omega$ such that $x$ lies in the closure of $|C|$, i.e. in

$$
\bigcup_{S \leq C}|S| \text {. }
$$

The vertices of $C$ can be given as

$$
e_{1} \rightarrow e_{2} \rightarrow \cdots \rightarrow e_{m} \rightarrow e_{1} .
$$

If $\left(\mu_{i}\right)$ are the barycentric coordinates of $x$ with respect to $\left(e_{i}\right)$, i.e.

$$
x=\sum_{i} \mu_{i} e_{i},
$$

then the class $\langle\mu\rangle$ is called the cyclic (simplicial) type of $x$ in $\Omega$.

Remark 6.2. 1. In general, not every edge has an orientation.

2. Definition 6.1 applies for $\mathcal{I}_{E_{D}}$ as well. The skew field is then $\mathrm{Z}_{D}\left(L_{\left[E_{D}: F\right]}\right)$ instead of $D$ and one has to substitute $d$ by $\frac{d}{\left[E_{D}: F\right]}$. The above chosen orientation is just the choice of one of the directions of rotation of the Coxeter diagram of $\Omega$.

Proposition 6.3. The notion of cyclic type does not depend on the choice of the chamber $C$ and the starting vertex $e_{1}$.

Proof: Let $x$ be a point of $\mathcal{I}$ contained in $|C|$ and $\left|C^{\prime}\right|$ for two chambers $C$ and $C^{\prime}$. Let $e_{1} \rightarrow e_{2} \rightarrow \cdots \rightarrow e_{m} \rightarrow e_{1}$ and $e_{1}^{\prime} \rightarrow e_{2}^{\prime} \rightarrow \cdots \rightarrow e_{m}^{\prime} \rightarrow e_{1}^{\prime}$ be the vertices of $C$ and $C^{\prime}$, respectively. It is clear that the cyclic type does not depend on the choice of the starting vertex $e_{1}$, and we can 
thus assume without loss of generality that $e_{1}=e_{1}^{\prime}$. Let $\left(\mu_{i}\right)$ be the barycentric coordinates of $x$ w.r.t. $\left(e_{i}\right)$. We have to show that they are also the barycentric coordinates w.r.t. $\left(e_{i}^{\prime}\right)$.

Let $\left(\mu_{i}^{\prime}\right)$ be the barycentric coordinates of $x$ w.r.t. $\left(e_{i}^{\prime}\right)$. By $e_{1}=e_{1}^{\prime}$, we have $\mu_{1}=\mu_{1}^{\prime}$. Without loss of generality let $\mu_{1}$ be non-zero. If $\mu_{1}=1$, then the other coordinates are zero and we are done. In case of $\mu_{1}<1$ let $i_{2}$ and $i_{2}^{\prime}$ be the first indexes greater than 1 , such that $\mu_{i_{2}}$ and $\mu_{i_{2}^{\prime}}^{\prime}$ are non-zero, respectively. Without loss of generality assume $i_{2}^{\prime} \leq i_{2}$. Let $j$ be the index such that $e_{j}$ is equal to $e_{i_{2}^{\prime}}^{\prime}$. Then $\mu_{j}$ is equal to $\mu_{i_{2}^{\prime}}^{\prime}$ and $j \geq i_{2}$ by the definition of $i_{2}$. Thus we have to prove that the inequalities

$$
i_{2}^{\prime} \leq i_{2} \leq j
$$

are equalities. Let $\mathcal{L}$ and $\mathcal{L}^{\prime}$ be lattice chains of $e_{1}$ and $e_{j}$, respectively, and choose $L \in \operatorname{im}(\mathcal{L})$ and $L^{\prime} \in \operatorname{im}\left(\mathcal{L}^{\prime}\right)$ such that $L \supset L^{\prime} \supset L \pi_{D}$. Then the $\kappa_{D}$-dimension of $L / L^{\prime}$ is equal to $j-1$ and $i_{2}^{\prime}-1$. Thus $j$ is equal to $i_{2}^{\prime}$. The result follows by induction.

We denote by $x_{\mathfrak{a}}$ the barycenter of $\mathfrak{a}$ in $\mathcal{I}$.

Theorem 6.4. Let $(E, \mathfrak{a})$ be an embedding of $A$ with embedding type $\langle\lambda\rangle$ and suppose $\mathfrak{a}$ has rank $r$. If $\langle\mu\rangle$ is the cyclic type of $j_{E_{D}}\left(x_{\mathfrak{a}}\right)$, then the following hold.

1. $\left[E_{D}: F\right] r \mu \in \mathbb{N}_{0}^{m}$, and

2. $\langle\operatorname{row}(\lambda)\rangle=\left\langle\left[E_{D}: F\right] r \mu\right\rangle^{c}$.

Remark 6.5. With Theorem 6.4 we can calculate the embedding type from the cyclic type. For example take $r=2, f=6, m=7$ and assume that $j_{E_{D}}\left(x_{\mathfrak{a}}\right)$ is

$$
\frac{3}{12} e_{1}+\frac{2}{12} e_{2}+\frac{1}{12} e_{3}+\frac{0}{12} e_{4}+\frac{0}{12} e_{5}+\frac{4}{12} e_{6}+\frac{2}{12} e_{7},
$$

and thus

$$
\operatorname{pairs}(\langle 12 \mu\rangle)=\operatorname{pairs}(\langle 3,2,1,0,0,4,2\rangle)=\langle(3,1),(2,1),(1,3),(4,1),(2,1)\rangle \text {. }
$$

From the dual

$$
\begin{aligned}
\langle 12 \mu\rangle^{c} & =\text { pairs }^{-1}(\langle(1,2),(1,1),(3,4),(1,2),(1,3)\rangle \\
& =\langle 1,0,1,3,0,0,0,1,0,1,0,0\rangle
\end{aligned}
$$


applying Theorem 6.4 we can deduce the embedding type of $(E, \mathfrak{a})$ :

$$
\left(\begin{array}{ll}
1 & 0 \\
1 & 3 \\
0 & 0 \\
0 & 1 \\
0 & 1 \\
0 & 0
\end{array}\right) .
$$

For the proof we can restrict to the case where $E=E_{D}$ because $(E, \mathfrak{a})$ is equivalent to $\left(E_{D}, \mathfrak{a}\right)$ and the statement of Theorem 6.4 just uses $E_{D}$ instead of $E$. We put $f:=[E: F]$, i.e.

$$
E \cong L_{f} \subseteq L
$$

and

$$
F \subseteq E \subseteq A_{E} \subseteq A
$$

Firstly we need some lemmas. The action of $G$ on square lattice functions by conjugation induces the following maps. For $g \in G$ we have:

1. $m_{g}: \Omega \rightarrow \Omega, \mathfrak{a} \mapsto g \mathfrak{a} g^{-1}$,

2. $\left|m_{g}\right|: \mathcal{I} \rightarrow \mathcal{I}$, where $\left|m_{g}\right|(x)$ is defined to be the element $z$ of $\mathcal{I}$, such that the square lattice function satisfies

$$
\mathfrak{g}_{z}(t)=g \mathfrak{g}_{x}(t) g^{-1}, \quad t \in \mathbb{R},
$$

and

3. $c_{g}: \mathcal{I}_{E} \rightarrow \mathcal{I}_{g E g^{-1}}$ defined via

$$
\mathfrak{g}_{c_{g}(y)}(t)=g \mathfrak{g}_{y}(t) g^{-1}, \quad t \in \mathbb{R},
$$

i.e. in terms of square lattice functions $c_{g}$ is a map from $\operatorname{Latt}_{o_{E}}^{2}\left(A_{E}\right)$ to Latt ${ }_{o_{g E g^{-1}}}^{2}\left(g A_{E} g^{-1}\right)$.

We say that a map between partially oriented graphs preserves orientations if an oriented edge is mapped to an oriented edge such that the direction is preserved.

Lemma 6.6. The maps $\left|m_{g}\right|$ and $c_{g}$ are affine bijections which induce orientation preserving isomorphisms on the simplicial structures of the Euclidean buildings. In particular, $m_{g}$ preserves the embedding type, $c_{g}$ the cyclic type, and the following diagram commutes:

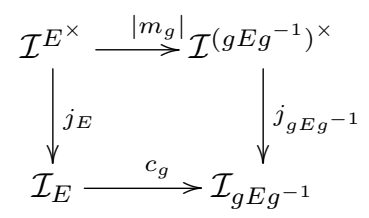


Proof: The lemma follows directly from the definitions of the maps involved.

The following lemma gives a geometric interpretation of the map

$\{$ cyclic matrices $\} \rightarrow\{$ cyclic matrices with only one column $\}$

$$
\langle\lambda\rangle \mapsto\left\langle\operatorname{row}(\lambda)^{T}\right\rangle .
$$

Lemma 6.7 (Rank reduction lemma). Assume there is a field extension $K \mid F$ of degree $s$ in $E \mid F$, where $2 \leq s \leq m$. Suppose $\mathfrak{a}$ is a vertex in $\Omega^{E^{\times}}$such that $\mathfrak{a} \cap \mathrm{Z}_{A}(K)$ is a facet of rank $s$ in $\Omega_{K}^{E^{\times}}$and assume $(E, \mathfrak{a})$ has embedding type $\langle\lambda\rangle$ and $\left(E, \mathfrak{a} \cap \mathrm{Z}_{K}(A)\right)$ has embedding type $\left\langle\lambda^{\prime}\right\rangle$. Then we get

$$
\operatorname{row}(\lambda) \sim \operatorname{row}\left(\lambda^{\prime}\right), \text { i.e. } \lambda \sim \operatorname{row}\left(\lambda^{\prime}\right)^{T} .
$$

Proof: By Lemma 6.6 it is enough to show the result only for one embedding equivalent to $(E, \mathfrak{a})$. For simplicity of exposition, we restrict ourselves to the case of $s=2$. The argument for $s>2$ is similar. We fix a $D$-basis of $V$. Then $(E, \mathfrak{a})$ is equivalent to the pearl embed$\operatorname{ding}\left(E_{\lambda}, \mathfrak{a}_{\lambda}\right)$ of $\lambda$, and moreover $\mathfrak{a}_{\lambda}$ is $\mathrm{M}_{m}\left(o_{D}\right)$. Now we apply a permutation $p$ on $\left(E_{\lambda}, \mathfrak{a}_{\lambda}\right)$ such that the odd exponents of $\sigma$ in $p E_{\lambda} p^{-1}$ are behind all even exponents, i.e. $p E_{\lambda} p^{-1}$ is the image of

$$
\begin{aligned}
& x \in L_{f} \mapsto \operatorname{diag}\left(M_{n_{1}}(x), M_{n_{2}}(x)\right), \quad n_{1}:=\sum_{i \text { odd }} \lambda_{i}, \quad n_{2}:=\sum_{i \text { even }} \lambda_{i} \\
& \text { where } M_{n_{1}}(x)=\operatorname{diag}\left(\sigma^{0}(x) \mathbb{1}_{\lambda_{1}}, \sigma^{2}(x) \mathbb{1}_{\lambda_{3}}, \ldots, \sigma^{f-2}(x) \mathbb{1}_{\lambda_{f-1}}\right) \\
& \quad \text { and } M_{n_{2}}(x)=\operatorname{diag}\left(\sigma^{1}(x) \mathbb{1}_{\lambda_{2}}, \sigma^{3}(x) \mathbb{1}_{\lambda_{4}}, \ldots, \sigma^{f-1}(x) \mathbb{1}_{\lambda_{f}}\right) .
\end{aligned}
$$

We conjugate $p\left(E_{\lambda}, \mathfrak{a}_{\lambda}\right) p^{-1}$ by the matrix $\operatorname{diag}\left(\mathbb{1}_{n_{1}}, \pi_{D}^{-1} \mathbb{1}_{n_{2}}\right)$ to obtain an embedding $\left(E^{\prime}, \mathfrak{a}^{\prime}\right)$ with the following properties. Let $K^{\prime} \mid F$ be the field extension of degree two in $E^{\prime} \mid F$.

- $K^{\prime}$ is the image of the diagonal embedding of $L_{2}$ in $\mathrm{M}_{m}(D)$ and its centralizer is $\mathrm{M}_{m}\left(D_{K^{\prime}}\right)$, where $D_{K^{\prime}}:=\mathrm{Z}_{D}\left(L_{2}\right)$. This follows because even powers of $\pi_{D}$ commute with $L_{2}$.

- The intersection of $\mathfrak{a}^{\prime}$ with $\mathrm{M}_{m}\left(D_{K^{\prime}}\right)$ is a hereditary order in standard form with invariant $\left\langle n_{1}, n_{2}\right\rangle$. The positivity of the integers $n_{i}$ follows from the assumption that this intersection is a facet of rank 2 .

Since $\pi_{D_{K^{\prime}}}:=\pi_{D}^{2}$ is a prime element of $D_{K^{\prime}}$ which normalizes $L$ and since the powers of $\sigma$ occurring in the description of $E^{\prime}$ are even we can 
read the embedding type of $\left(E^{\prime}, \mathfrak{a}^{\prime} \cap \mathrm{M}_{m}\left(D_{K^{\prime}}\right)\right)$ directly. It is the class of

Thus the result follows.

$$
\left(\begin{array}{cc}
\lambda_{1} & \lambda_{2} \\
\lambda_{3} & \lambda_{4} \\
\vdots & \vdots \\
\lambda_{f-1} & \lambda_{f}
\end{array}\right)
$$

The next lemma shows that changing the skew field does not change the embedding type.

Lemma 6.8 (Changing skew field lemma). Let $D^{\prime}$ be a skew field central and of finite index $d$ over a non-Archimedean local field $F^{\prime}$. Suppose further that $L^{\prime} \mid F^{\prime}$ is a maximal unramified field extension in $D^{\prime} \mid F^{\prime}$ normalized by a uniformizer $\pi_{D^{\prime}}$ of $D^{\prime}$. Let $V^{\prime}$ be an m-dimensional right $D^{\prime}$-vector space. Denote the Euclidean building of $\mathrm{GL}_{m}\left(D^{\prime}\right)$ by $\Omega^{\prime}$ and let $\Sigma, \Sigma^{\prime}$ be the apartments of $\Omega, \Omega^{\prime}$ corresponding to the standard bases $\left(v_{i}\right),\left(v_{i}^{\prime}\right)$, respectively. Then $\Sigma^{\prime}$ is fixed by the image $E^{\prime}$ of the $d i$ agonal embedding of $L_{f}^{\prime}$ in $\mathrm{M}_{m}\left(D^{\prime}\right)$. Assume further that $E$ is the image of the diagonal embedding of $L_{f}$ in $\mathrm{M}_{m}(D)$. Under these assumptions the map defined by

$$
\left[t \mapsto \bigoplus_{i} v_{i} \mathfrak{p}_{D}^{\left[d\left(t+\alpha_{i}\right)\right]+}\right] \mapsto\left[t \mapsto \bigoplus_{i} v_{i}^{\prime} \mathfrak{p}_{D^{\prime}}^{\left[d\left(t+\alpha_{i}\right)\right]+}\right]
$$

from $|\Sigma|$ to $\left|\Sigma^{\prime}\right|$ is the geometric realization $|\phi|$ of an isomorphism $\phi$ of simplicial complexes which preserves orientations and embedding types. The latter means that if $\mathfrak{a}^{\prime}$ is the image of a hereditary order $\mathfrak{a}$ under $\phi$ then the embedding types of $(E, \mathfrak{a})$ and $\left(E^{\prime}, \mathfrak{a}^{\prime}\right)$ equal.

We want to remark that there is no condition about how $F$ is related to $F^{\prime}$, so they could have different residue characteristics, but the map $\phi$ in the statement is of course only a map between apartments, and not between buildings.

Proof: We define $\phi$ to map the class of a lattice chain $\mathfrak{L}$ with

$$
\mathfrak{L}_{j}=\bigoplus_{i} v_{i} \mathfrak{p}_{D}^{\nu_{i, j}}
$$

to the class of $\mathfrak{L}^{\prime}$ with

$$
\mathfrak{L}_{j}^{\prime}=\bigoplus_{i} v_{i}^{\prime} \mathfrak{p}_{D^{\prime}}^{\nu_{i, j}}
$$

We only show that the embedding type is preserved. The other properties are verified easily. We take the two lattice chains $\mathfrak{L}$ and $\mathfrak{L}^{\prime}$ with 
corresponding hereditary orders $\mathfrak{a}$ and $\mathfrak{a}^{\prime}$. Applying from the left an appropriative permutation matrix $P$ and an appropriative diagonal matrix $T$ (resp. $T^{\prime}$ ), whose non-zero entries are powers of the corresponding prime element, we obtain simultaneously lattice chains corresponding to hereditary orders $\mathfrak{b}, \mathfrak{b}^{\prime}$ in the same standard form. More precisely $T^{\prime}$ is obtained from $T$ by substituting $\pi_{D^{\prime}}$ for $\pi_{D}$. Thus, $\left(T P E P^{-1} T^{-1}, \mathfrak{b}\right)$ and $\left(T^{\prime} P E^{\prime} P^{-1} T^{\prime-1}, \mathfrak{b}^{\prime}\right)$ have the same embedding type, and thus, by conjugating back, $(E, \mathfrak{a})$ and $\left(E^{\prime}, \mathfrak{a}^{\prime}\right)$ have the same embedding type.

We now fix a $D$-basis $v_{1}, \ldots, v_{m}$ of $V$ and therefore a frame

$$
\mathcal{R}:=\left\{v_{i} D \mid 1 \leq i \leq m\right\}
$$

and $\Sigma=\Omega_{\mathcal{R}}$ the apartment of $\Omega$ corresponding to $\mathcal{R}$. The algebra $A$ is identified with $\mathrm{M}_{m}(D)$. By the affine bijection $|\Sigma| \cong \mathbb{R}^{m-1}$ which maps

$$
[\Lambda] \text { with } \Lambda(t)=\bigoplus_{i} \mathfrak{p}_{D}^{\left[\left(t+\alpha_{i}\right) d\right]+} \text { to }\left(\left(\alpha_{1}-\alpha_{2}\right) d, \ldots,\left(\alpha_{m-1}-\alpha_{m}\right) d\right)
$$

we can introduce affine coordinates on $|\Sigma|$. We denote the points of $|\Sigma|$ corresponding to the vectors $0,(f, 0, \ldots, 0),(0, f, 0, \ldots, 0), \ldots,(0, \ldots, 0, f)$ by $Q_{1}, Q_{2}, \ldots, Q_{m}$.

Remark 6.9. The vertices of $\Sigma$ are exactly the simplices corresponding to the points of

$$
Q_{1}+\sum_{i=2}^{m} \frac{1}{f} \mathbb{Z}\left(Q_{i}-Q_{1}\right)
$$

Remark 6.10. For an element $g \in \cap_{i=1}^{m}\left(\operatorname{End}_{D}\left(v_{i} D\right)\right)^{\times}$, i.e. a diagonal matrix, the map $\left|m_{g}\right|$ induces an affine bijection of $|\Sigma|$. If $g$ is $\operatorname{diag}\left(1, \ldots, 1, \pi_{D}^{k}, 1, \ldots, 1\right)$, with $\pi_{D}^{k}$ in the $i$-th row, then $\left|m_{g}\right|$ is of the form

$$
x \mapsto x+\frac{k}{f}\left(Q_{i+1}-Q_{i}\right),
$$

where $Q_{m+1}$ is understood to mean $Q_{1}$. To prove this statement it is enough to prove it for elements $x$ of $|\Sigma|$ corresponding to vertices and group elements $g$ of the above simple form with $k=1$. The latter is an easy calculation using a lattice chain corresponding to $x$.

Example 6.11. Let us assume $E$ is the image of the diagonal embedding of $L_{f}$ in $\mathrm{M}_{m}(D)$, i.e.

$$
E=\left\{\operatorname{diag}(x, \ldots, x) \mid x \in L_{f}\right\} .
$$

Then $A_{E}$ and $j_{E}$ simplify, i.e.

1. $A_{E} \cong \operatorname{End}_{D_{E}}(W)$ with $D_{E}:=\mathrm{Z}_{D}\left(L_{f}\right)$ and $W:=\bigoplus_{i} v_{i} D_{E}$. 
2. The geometric realization of $\Sigma$ is a subset of $\mathcal{I}^{E^{\times}}$.

3. For $[\Lambda] \in \mathcal{I}$ we have

$$
j_{E}([\Lambda])=[\Lambda \cap W],
$$

where $\Lambda \cap W$ denotes the lattice function

$$
x \mapsto \Lambda(x) \cap W .
$$

4. The image of $\left.j_{E}\right|_{|\Sigma|}$ is the geometric realization of the apartment $\Sigma_{E}$ which belongs to the frame $\left\{v_{i} D_{E} \mid 1 \leq i \leq m\right\}$ and in affine coordinates the map has the form

$$
x \in \mathbb{R}^{m-1} \mapsto \frac{1}{f} x \in \mathbb{R}^{m-1} .
$$

5. The vertices of $\Sigma_{E}$ are the points of $\left|\Sigma_{E}\right|$ with affine coordinate vectors in $\mathbb{Z}^{m-1}$. Specifically the points $P_{i}:=j_{E}\left(Q_{i}\right)$ are vertices of a chamber of $\Sigma_{E}$.

6. The edge between $P_{i}$ and $P_{i+1}$ is oriented towards $P_{i+1}$.

Proof of Example 6.11: The statement 1 is trivial and 5 and 6 follow from 4.

For 2: We have $|\Sigma| \subseteq \mathcal{I}^{E^{\times}}$because, for an $o_{D}$-lattice function $\Lambda$ split by $\mathcal{R}$, the action of an element of $E^{\times}$on $\Lambda$ is the multiplication of every lattice $\Lambda(t)$ by a fixed element $x \in D^{\times}$.

For 3 and 4: We use the decomposition

$$
V=W \otimes_{D_{E}} D=W \oplus W \pi_{D} \oplus W \pi_{D}^{2} \oplus \cdots \oplus W \pi_{D}^{f-1} .
$$

The function $\mathcal{I}_{E} \rightarrow \mathcal{I}$ which maps $[\Gamma] \in \mathcal{I}_{E}$ to $[\Lambda] \in \mathcal{I}$ defined by

$$
\Lambda(t):=\bigoplus_{i=0}^{f-1} \Gamma\left(t-\frac{i}{d}\right) \pi_{D}^{i},
$$

is affine and $A_{E}^{\times}$-equivariant. By Theorem 5.2 it has to be $j_{E}^{-1}$ and thus $j_{E}([\Lambda])$ is equal to $[\Lambda \cap W]$. The appearance of $j_{E}$ in terms of coordinates follows now from

for $t \in \mathbb{R}$.

$$
\mathfrak{p}_{D}^{[t]+} \cap D_{E}=\mathfrak{p}_{D_{E}}^{\left[\frac{t}{f}\right]+},
$$

Proof of Theorem 6.4: By Lemma 6.6 and Theorem 4.4 we can assume that we are in the situation of Example 6.11 above and that there is a diagonal matrix $h$ consisting of powers of $\pi_{D}$ with non-negative exponents less than $f$ such that

$$
\left(h E h^{-1}, h \mathfrak{a} h^{-1}\right)
$$

is the pearl embedding of $\lambda$. We consider two cases for the proof. 
Case 1: a has rank 1, i.e.

$$
h \mathfrak{a} h^{-1}=\mathrm{M}_{m}\left(o_{D}\right)=\mathfrak{a}_{Q_{1}},
$$

and $\lambda$ has only one column. We get $x_{\mathfrak{a}}$ from $Q_{1}$ by applying $m_{h^{-1}}$ which is a composition of maps $m_{g}$ where $g$ differs from the identity matrix by only one diagonal entry $\pi_{D}^{k}$. Now Remark 6.10 gives

$$
x_{\mathfrak{a}}=Q_{1}-\sum_{j=1}^{m} \frac{a_{j}}{f}\left(Q_{j+1}-Q_{j}\right),
$$

where $a_{j}=k-1$ if

$$
\sum_{i=1}^{k-1} \lambda_{i}<j \leq \sum_{i=1}^{k} \lambda_{i}
$$

Thus in barycentric coordinates $j_{E}\left(x_{\mathfrak{a}}\right)$ has the form

$$
\frac{f-a_{m}+a_{1}}{f} P_{1}+\frac{a_{2}-a_{1}}{f} P_{2}+\cdots+\frac{a_{m}-a_{m-1}}{f} P_{m},
$$

and therefore

$$
\mu:=\left(\frac{f-a_{m}+a_{1}}{f}, \frac{a_{2}-a_{1}}{f}, \ldots, \frac{a_{m}-a_{m-1}}{f}\right)
$$

satisfies part 1 of the theorem. If $\left(\lambda_{i_{l}}\right)_{1 \leq l \leq s}$ is the subsequence of nonzero entries we define the indices

$$
j_{l}:=\lambda_{i_{1}}+\lambda_{i_{2}}+\cdots+\lambda_{i_{l-1}}+1, \quad 2 \leq l \leq s,
$$

and $j_{1}:=1$. This are the indices where the $\mu_{j}$ are non-zero, more precisely from

$$
j_{l}=\sum_{i=1}^{i_{l}-1} \lambda_{i}+1 \leq \sum_{i=1}^{i_{l}} \lambda_{i}
$$

we obtain for $a_{j}$ the following values:

$a_{j}=a_{j_{l}}=i_{l}-1, j_{l} \leq j<j_{l+1}, \quad$ and $\quad a_{j}=a_{j_{s}}=i_{s}-1, j_{s} \leq j \leq m$, and thus the subsequence of non-zero entries of $f \mu$ is

$$
\left(f \mu_{j_{l}}\right)=\left(f-i_{s}+i_{1}, i_{2}-i_{1}, i_{3}-i_{2}, \ldots, i_{s}-i_{s-1}\right) .
$$

Therefore pairs $(\langle f \mu\rangle)$ is equal to

$$
\left\langle\left(f-i_{s}+i_{1}, \lambda_{i_{1}}\right),\left(i_{2}-i_{1}, \lambda_{i_{2}}\right),\left(i_{3}-i_{2}, \lambda_{i_{3}}\right), \ldots,\left(i_{s}-i_{s-1}, \lambda_{i_{s}}\right)\right\rangle
$$

and this is precisely pairs $\left(\langle\operatorname{row}(\lambda)\rangle^{c}\right)$.

Case 2: Assume the rank $r$ of $\mathfrak{a}$ is not 1. Here we want to use rank reduction. We fix an unramified field extension $L^{\prime} \mid F$ of degree $r d$ in an algebraic closure of $F$. Denote by $D^{\prime}$ a skew field which is a central 
cyclic algebra over $F$ with maximal field $L^{\prime}$ and an $L^{\prime}$-normalizing prime element $\pi_{D^{\prime}}$, i.e.

$$
D^{\prime}=\bigoplus_{i=0}^{d r-1} L^{\prime} \pi_{D^{\prime}}^{i}, \quad \pi_{D^{\prime}} L^{\prime} \pi_{D^{\prime}}^{-1}=L^{\prime}, \quad \text { and } \quad \pi_{D^{\prime}}^{d r}=\pi_{F}
$$

The images of $L_{r}^{\prime}, L_{r f}^{\prime}$ under the diagonal embedding of $L^{\prime}$ in $\mathrm{M}_{m}\left(o_{D^{\prime}}\right)$ are denoted by $F^{\prime}, E^{\prime}$, respectively, and the apartment of the Euclidean building $\Omega^{\prime}$ of $\mathrm{GL}_{m}\left(D^{\prime}\right)$ corresponding to the standard basis is denoted by $\Sigma^{\prime}$, i.e. we have a field tower

$$
E^{\prime} \supseteq F^{\prime} \supseteq F,
$$

apartments $\Sigma_{E^{\prime}}^{\prime}, \Sigma_{F^{\prime}}^{\prime}, \Sigma^{\prime}$ of $\Omega_{E^{\prime}}^{\prime}, \Omega_{F^{\prime}}^{\prime}, \Omega^{\prime}$ and reduced Bruhat-Tits buildings $\mathcal{I}_{E^{\prime}}^{\prime}, \mathcal{I}_{F^{\prime}}^{\prime}, \mathcal{I}^{\prime}$, respectively. We then obtain a commutative diagram of bijections, where the lines are induced by isomorphisms of chamber complexes which preserve orientations,

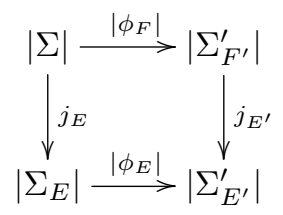

The map $\left|\phi_{F}\right|$ is given by

$$
\left[x \mapsto \bigoplus_{i=0}^{m-1} v_{i} \mathfrak{p}_{D}^{\left.\left[d\left(x+\alpha_{i}\right)\right]+\right]}\right]\left[x \mapsto \bigoplus_{i=0}^{m-1} v_{i}^{\prime} \mathfrak{p}_{\mathrm{Z}_{D^{\prime}}\left(L_{r}^{\prime}\right)}^{\left[d\left(x+\alpha_{i}\right)\right]+}\right]
$$

and $\left|\phi_{E}\right|$ analogously. Here $\left(v_{i}^{\prime}\right)$ is the standard basis of $D^{\prime m}$. Because of Lemma 6.8, the map $\left|\phi_{F}\right|$ preserves the embedding type and thus we can finish the proof by applying Lemma 6.7 to

$$
\Sigma^{\prime} \rightarrow \Sigma_{F^{\prime}}^{\prime} \rightarrow \Sigma_{E^{\prime}}^{\prime}
$$

More precisely, $\phi_{F}(\mathfrak{a})$ is a facet of rank $r$ in $\Sigma_{F^{\prime}}^{\prime}$. Its barycenter has affine coordinates in $\frac{1}{r} \mathbb{Z}^{m-1}$ and therefore its preimage under $j_{F^{\prime}}$ is a point $y$ with integer affine coefficients, i.e. it corresponds to a vertex of $\Omega^{\prime}$. To emphasize the base field we write field extensions as the index of $j$. Because

$$
\left.j_{E^{\prime} \mid F^{\prime}}\left(x_{\phi_{F}(\mathfrak{a})}\right)\right)=j_{E^{\prime} \mid F^{\prime}}\left(j_{F^{\prime} \mid F}(y)\right)=j_{E^{\prime} \mid F}(y),
$$

the theorem follows now from the rank reduction lemma and Case 1. 


\section{References}

[BG00] P. Broussous and M. Grabitz, Pure elements and intertwining classes of simple strata in local central simple algebras, Comm. Algebra 28(11) (2000), 5405-5442. DOI: 10.1080/ 00927870008827164.

[BL02] P. Broussous and B. Lemaire, Building of $G L(m, D)$ and centralizers, Transform. Groups 7(1) (2002), 15-50. DOI: 10.1007/s00031-002-0002-5.

[BSS12] P. Broussous, V. SÉcherre, and S. Stevens, Smooth representations of $\mathrm{GL}_{m}(D)$ V: Endo-classes, Doc. Math. 17 (2012), $23-77$.

[BT84] F. Bruhat And J. Tits, Schémas en groupes et immeubles des groupes classiques sur un corps local, Bull. Soc. Math. France 112(2) (1984), 259-301.

[Frö87] A. FröHLICH, Principal orders and embedding of local fields in algebras, Proc. London Math. Soc. (3) 54(2) (1987), 247-266. DOI: $10.1112 / \mathrm{plms} / \mathrm{s} 3-54.2 .247$.

[Gra99] M. Grabitz, Continuation of hereditary orders in local central simple algebras, J. Number Theory 77(1) (1999), 1-26. DOI: 10.1006/jnth.1999.2374.

[MP94] A. Moy and G. Prasad, Unrefined minimal $K$-types for p-adic groups, Invent. Math. 116(1-3) (1994), 393-408. DOI: 10.1007/BF01231566.

[Rei03] I. ReINER, "Maximal orders", Corrected reprint of the 1975 original, With a foreword by M. J. Taylor, London Mathematical Society Monographs. New Series 28, The Clarendon Press, Oxford University Press, Oxford, 2003.

Mathematisches Institut

Universität Münster

Einsteinstraße 62

48149 Münster

Deutschland

E-mail address: dskod_01@uni-muenster.de

Primera versió rebuda el 30 de juliol de 2013, darrera versió rebuda el 10 de març de 2014. 